\title{
Muons in minimum bias events from the first CMS data
}

\author{
Lucia Barbone (for the CMS Collaboration) \\ Dipartimento Interateneo di Fisica and INFN Sezione di Bari, via E. Orabona 4, 70126 Bari, Italy
}

\begin{abstract}
Muon signatures are extremely important in the challenging environment of collisions at the LHC. Understanding the observed muon yield from Standard Model processes is the first task for all analyses involving muons. Basic muon kinematical observables are presented for the first CMS data at $\sqrt{s}=7 \mathrm{TeV}$ and compared with Monte Carlo expectations. The muon sample composition is studied by using the MC truth in terms of muons from heavy flavours, muons from light hadron decays and hadron punch-through is studied from MC truth.
\end{abstract}

Keywords: Particle detectors, proton-proton collisions, LHC, CMS, muons.

PACS: 24.10.Lx, 29.40.Ka, 29.85.-c, 29.85.Fj

\section{INTRODUCTION}

Many of the rare physics processes searched for in hadron collider events are characterized by leptons in the final state. Muons provide a striking signature in a large background of jets, allowing for the selection of rare signal events. Thus it is crucial for CMS [1] to be able to detect muons with high efficiency, low fake-rate from backgrounds and excellent momentum resolution.

Muon reconstruction in CMS and the response of various subdetectors to muons have been studied in great detail using muons from cosmic rays [2]. Here we compare data and Monte Carlo and study in particular the expected composition of the muon sample. The results are obtained using minimum bias data sample collected in early spring 2010 at $\sqrt{s}=7 \mathrm{TeV}$, corresponding to an integrated luminosity of about $0.19 n b^{-1}$.

\section{MUON RECONSTRUCTION IN CMS}

Muon tracking in CMS is performed with the all-silicon tracker at the heart of the detector and with up to four stations of gas-ionization muon detectors [3] installed outside the solenoid, sandwiched between steel layers serving both as hadron absorbers and as a return yoke for the magnetic field. Drift Tube (DT) chambers and Cathode Strip Chambers (CSC) are used in the barrel $(|\eta|<1.2)$ and endcaps $(0.9<|\eta|<2.4)$ respectively, complemented by a system of Resistive Plate Chambers (RPC) covering both regions (up to $|\eta|<1.6$ ). A cross-section of CMS is shown in Fig. 1.

In the standard CMS muon reconstruction [4], tracks are first reconstructed independently in the silicon tracker (tracker track) and in the muon spectrometer (standalonemuon track). On this basis, two reconstruction approaches are used:

- Global Muon reconstruction (outside-in): starting from a standalone-muon track in

CP1317, QCD@WORK 2010, International Workshop on Quantum Chromodynamics: Theory and Experiment edited by L. Angelini, G. E. Bruno, P. Colangelo, D. Creanza, F. De Fazio, and E. Nappi

(C) 2010 American Institute of Physics 978-0-7354-0872-2/10/\$30.00 

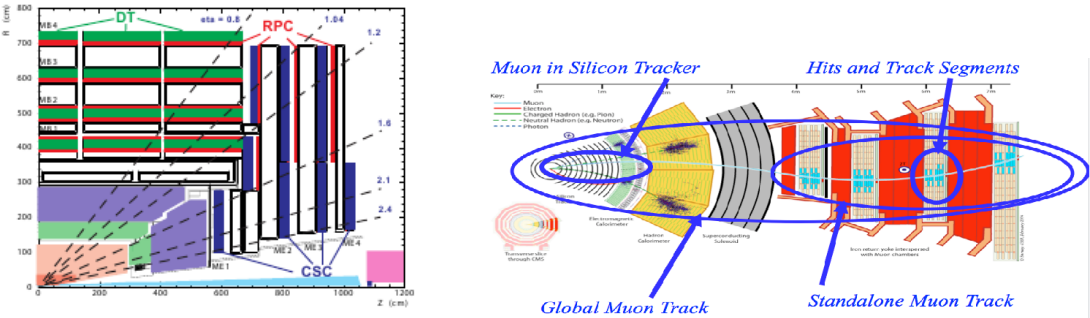

FIGURE 1. Left: Layout of one quadrant of CMS; Right: Types of muon candidates

the muon system, a matching tracker track is selected and a global-muon is fitted combining hits from the tracker track and standalone-muon track.

- Tracker Muon reconstruction (inside-out): all tracker tracks with $p_{T}>0.5 \mathrm{GeV} / \mathrm{c}$ and $p>2.5 \mathrm{GeV} / \mathrm{c}$ are extrapolated to the muon system, taking into account the expected energy loss and the uncertainty due to multiple scattering. If at least one muon segment (i.e. a short track stub made of DT or CSC hits) matches the extrapolated track in position, the corresponding tracker track qualifies as a trackermиол.

At low momentum (roughly $p<5 \mathrm{GeV} / \mathrm{c}$ ) the second approach is more efficient than the global muon reconstruction, since it requires only a single muon segment in the muon system, while global muon reconstruction typically becomes efficient with two or more segments. The majority of muons from collisions (with enough momentum) are reconstructed either as a Global Muon or a Tracker Muon, or often as both. However, if both approaches fail and only a standalone-muon track is found, this leads to a third category of muon candidates:

- Standalone-muon: this occurs only for about $1 \%$ of muons from collisions, thanks to the high tracker-track efficiency [5].

The results of these three algorithms are merged into a single collection of muon candidates, each one containing informations from the standalone, tracker and global fit, when available. A graphical description of the three types of reconstructed muons is shown in Fig. 1.

In this study the performances of the first two types are investigated. In Tracker Muon reconstruction, due to the low- $p_{T}$ cutoff and the higher fake rate, additional muonID requirements are applied. A matching segment is required in the outermost station where a segment is expected from the track extrapolation, matching both in position and direction with the expectation.

\section{KINEMATICAL DISTRIBUTIONS}

In this section we present kinematical distributions of a fully inclusive sample of muons reconstructed in minimum-bias collision events. An amount of 110,000 events have been preselected in the used data sample. Only good runs or run portions have been 
included after application of minimum bias technical trigger and the official preselection, requiring stable beams and all detectors in nominal operational status. Cosmics and beam-gas background are removed efficiently by requiring a good primary vertex in the event and by vetoing "beam scraping" events.

For the simulation we use 10.9 million minimum bias events generated with PYTHIA 6.422 with the D6T tune, filtered from a full sample corresponding to about $0.15 n b^{-1}$ of integrated luminosity. Standard preselection of minimum-bias events is applied in simulation as well.

\section{Monte Carlo Truth composition}

Each reconstructed muon in simulated events is matched unambiguously to a simulated particle, by using the MuonAssociatorByHits, a standard MC Truth tool that implements matching at the level of reconstructed and simulated hits. This tool provides the true association without any limitations of alternative geometric criteria. After checking the set of hits owned by a reconstructed muon track, this is associated to the simulated track which shares the highest number of hits with it. Only the hits in the muon detectors are used to define the association. The true particle originating those hits is determined. When this is a muon, the parent particle is searched to discriminate light and heavy flavour origin. This allows the classification of reconstructed muons into the following categories:

- Muons from heavy flavour: the majority of muon chamber hits associated to the muon candidate are produced by a true muon, whose parent particle can be a beauty meson, a charmed meson, a tau lepton or any other particle (like $\mathrm{W}$ or Z) not included in the "Light Flavour" category.

- Muons from light flavour: the majority of muon chamber hits of the muon candidate are produced by a true muon, which is originated from light hadron decays ( $\pi$ and $\mathrm{K}$ ) or, less frequently, inside a calorimeter shower or as decay of a product of a nuclear interaction in the detector material.

- Hadronic punch-through: the majority of muon chamber hits of the muon candidate are produced by a particle other than a muon. This component is generated in hadronic showers, although it includes a small fraction of primary hadrons (socalled "sail-through").

- Ghost or Duplicate: if one simulated particle gives rise to more than one reconstructed muon candidate, the one with the largest number of matched hits is flagged according to one of the previous categories, whereas any other is labelled as "duplicate" or "ghost". These are created by instrumental effects or imperfections in the pattern recognition of the reconstruction software. 
TABLE 1. Composition of the sources of reconstructed muons in minimum bias events according to simulation.

\begin{tabular}{|l|c|c|}
\hline & Global Muons & Tracker Muons \\
\hline Muons from heavy flavours & $23.9 \%$ & $8.8 \%$ \\
\hline Muons from light hadrons & $73.6 \%$ & $83.9 \%$ \\
\hline Hadronic punch-through & $1.8 \%$ & $4.5 \%$ \\
\hline Duplicates & $0.7 \%$ & $2.8 \%$ \\
\hline
\end{tabular}

\section{Results}

The inclusive muon distributions in events filtered by minimum bias trigger agree well in shape with the PYTHIA Minimum Bias Monte Carlo predictions both for Tracker and Global muons without being any specific selection applied. A selection of plots is presented to provide the comparison of Global Muon and Tracker Muon quantities, with simulated data normalized to the number of muons in the data.

There are about 18,000 Global Muons reconstructed in data and about 15,000 in the MC sample, while there are about 90,000 Tracker Muons reconstructed in data and about 76,000 in Monte Carlo. Table 1 lists the composition of Global Muons and Tracker Muons according to MC truth in minimum-bias events. The majority of reconstructed muon candidates originate from decays in flight of pions and kaons ("light flavour"). The rate of punch-through and fake muons is $2.5 \%$ for Global Muons and $7.3 \%$ for Tracker Muons, according to the Monte Carlo simulation.

The $\eta$ distributions (Fig. 2) of both Global and Tracker Muons peak in the forward region because there the minimum $p_{T}$ required to reach the muon stations is lower than in the barrel. The $p_{T}$ threshold is about $3 \mathrm{GeV} / \mathrm{c}$ in the barrel and about $0.5 \mathrm{GeV} / \mathrm{c}$ in the endcaps. In general the agreement between data and predictions from the simulation is good over the whole accessible range. Most of the muons going in the endcaps come from decays in flight of charged pions and kaons, while the fraction of muons from heavy flavour decays is relatively higher in the barrel region.

The $p_{T}$ and $p$ distributions are shown in Fig. 3 and Fig. 4. It can be appreciated that the fraction of muons with heavy flavour origin increases with increasing momentum values.

The transverse impact parameter distribution is shown in Fig. 5. As expected, muons from heavy flavours are limited to impact parameters below about $1 \mathrm{~mm}$, while the tails are completely due to non prompt muons from light hadron decays.

The overall data/MC agreement is quite good in shape. Moreover the agreement holds also for the absolute normalization, which is found to be within $3 \%$, well within the quoted uncertainty on the luminosity [6].

\section{CONCLUSION}

Muon signatures are extremely important in the challenging environment of protonproton collisions at the LHC. Understanding the observed muon yield from Standard 

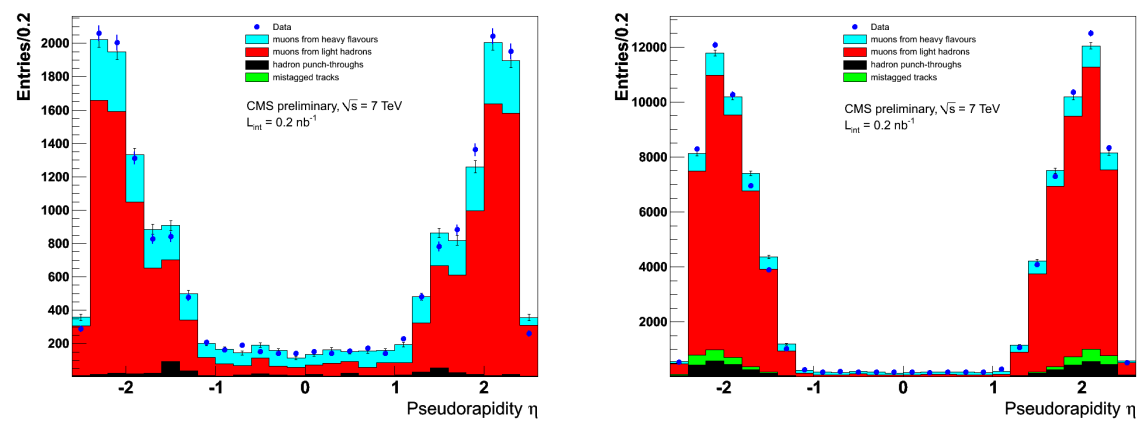

FIGURE 2. Pseudo-rapidity distribution of muons reconstructed in minimum bias data, compared with Monte Carlo expectations, normalized to the data, for Global Muons (left) and for Tracker Muons (right). The MC composition is shown by the stacked coloured histograms.
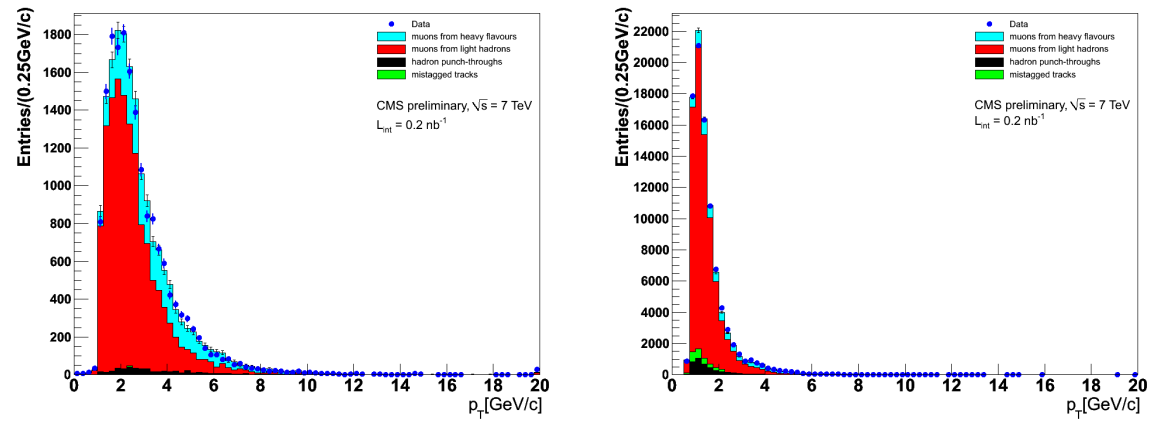

FIGURE 3. Muon transverse momentum distributions in minimum bias data, compared with Monte Carlo expectations, normalized to the data for Global Muons (left) and for Tracker Muons (right). The MC composition is shown by the stacked coloured histograms.

Model processes is the first task for all analyses including muons. A preliminary CMS study of muons in Minimum Bias data - presented here - show a remarkably good agreement with MC expectations. This result was not taken for granted given the extended inclusive nature of the considered sample of muons and represents a reference baseline for those forthcoming analyses concerning the heavy flavour production to be estimated at the LHC energies. 

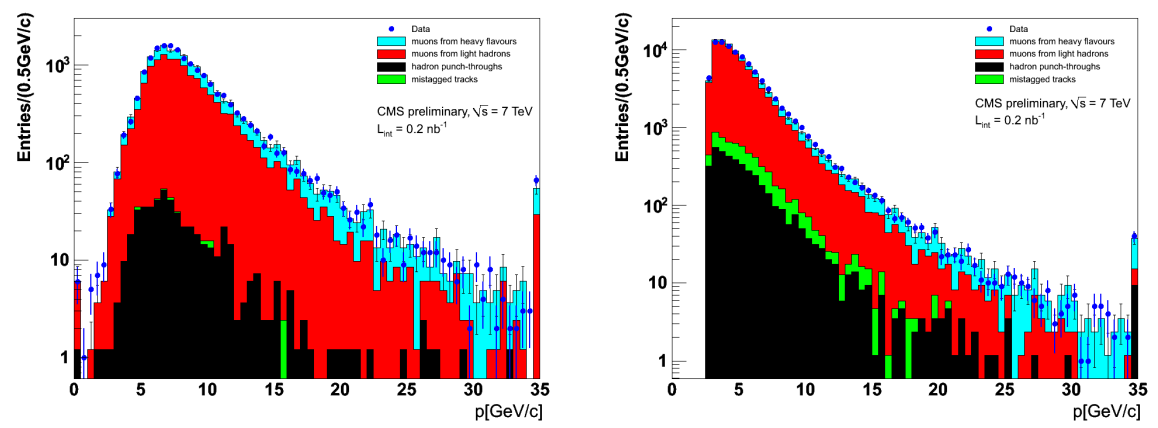

FIGURE 4. Muon momentum distributions in minimum bias data, compared with Monte Carlo expectations, normalized to the data for Global Muons (left) and for Tracker Muons (right). The MC composition is shown by the stacked coloured histograms.
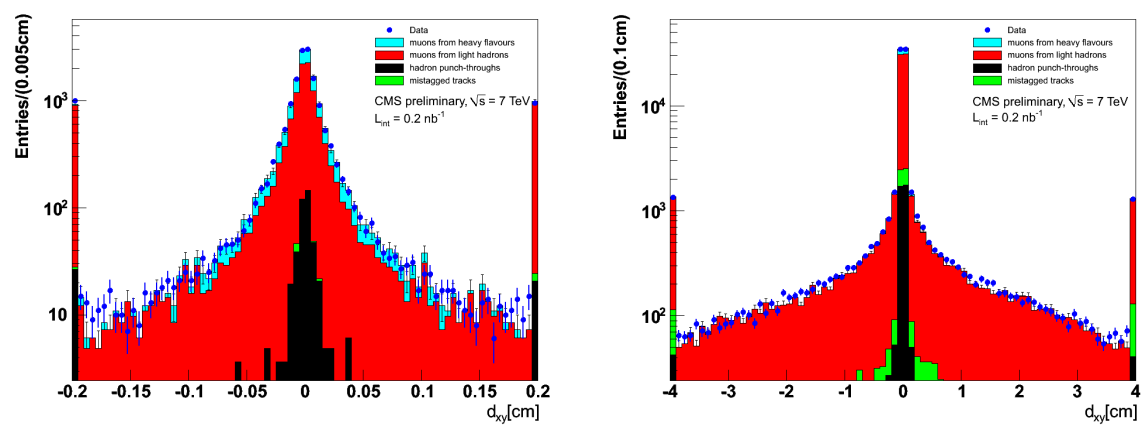

FIGURE 5. Muon transverse impact parameter with respect to the primary vertex in minimum bias data, compared with Monte Carlo expectations, normalized to the data for Global Muons (left) and for Tracker Muons (right). The MC composition is shown by the stacked coloured histograms.

\section{REFERENCES}

1. CMS Collaboration, "The CMS Experiment at the CERN LHC", JINST 3 (2008) S08004.

2. CMS Collaboration, "Performance of CMS muon reconstruction in cosmic-ray events", JINST 5 (2010) T03022.

3. CMS Collaboration, "CMS Muon Technical Design Report", CERN/LHCC 1997-032 (1997).

4. CMS Collaboration, "CMS Physics TDR: Volume 1, Detector Performance and Software", CERN/LHCC 2006-001 (2006).

5. CMS Collaboration, "Measurement of Tracking Efficiency", CMS PAS TRK-10-002 (2010).

6. CMS Collaboration, "Performance of muon identification in pp collisions at $\sqrt{s}=7$ TeV", CMS PAS MUO-10-002. 